\title{
A QoS Architecture Integrating Ad-Hoc and Infrastructure in Next Generation Networks
}

\author{
Marek Natkaniec ${ }^{1}$, Janusz Gozdecki ${ }^{1}$, Susana Sargento ${ }^{2}$ \\ ' Department of Telecommunications AGH University of Science and Technology, \\ Al. Mickiewicza 30, Cracow, Poland \\ ${ }^{2}$ Instituto de Telecomunicações, Universidade de Aveiro, Portugal \\ \{natkaniec(@kt.agh.edu.pl, gozdecki@kt.agh.edu.pl, ssargento@det.ua.pt\}
}

\begin{abstract}
This paper proposes the complete QoS architecture for integration of ad-hoc with infrastructure networks. The technology, service differentiation mechanisms, and signaling protocols are discussed. The modules required in the network elements and its integration to provide end-to-end QoS in mobile adhoc networks are presented. The proposed solution is based on the SWAN model with some extensions to provide $\mathrm{L} 2$ differentiation for four traffic classes and supports the integration with infrastructure networks. The deployed hierarchical architecture guarantees scalability and make possible per-flow resource management in wireless access where scarce radio resources should be managed effectively, and per-aggregate traffic management using a DiffServ model in the core.
\end{abstract}

Keywords: Ad-hoc networks, Infrastructure networks, Quality of Service, Integration, Service Differentiation, Signaling protocols

\section{Introduction}

Wireless Local Area Networks (WLANs) are one of the fastest growing areas of modern telecommunications today. They can be installed in places that are very difficult to wire as, for example, trading floors, manufacturing facilities, warehouses or historical buildings. WLANs are being widely implemented in many venues from markets and airports to retail, manufacturing, hospitals and corporate environments; they are beginning to be available in public spaces such as schools, hotels, restaurants, malls and shops. This technology offers the highest level of performance and capability features among other local wireless solutions. WLANs play a very important role in the network architecture as a provider of easy and unconstrained access to the wired infrastructure. Currently ad-hoc networking is becoming a promising solution to increase the radio coverage of broadband wireless systems, extending coverage of hotspots. This business strategy is profitable for both the provider and the user. Since a radio range is strongly affected in closed spaces or in areas with dense radio interferences, the resilience provoked by the multi-hop characteristics of mobile ad-hoc networks makes these especially appropriate to 
provide increased radio coverage with low cost and easy deployment. Therefore, adhoc networks play an increasing role in network access.

Ad-hoc networks differ clearly from the traditional cable infrastructure. This kind of networks is characterized by very dynamic changes of topologies and hence their design requires special attention. To support the present users and service requirements, the ad-hoc network needs to support differentiated QoS, which is a major challenge. The protocols assuring QoS in ad-hoc networks need to operate in a distributed way along the ad-hoc nodes, with proper mechanisms for reacting in a responsive way to any changes (e.g., topology, new sessions, congestion). There are some solutions for QoS support in ad-hoc networks: SWAN (Stateless Wireless Adhoc Networks) [4], INSIGNIA [6], FQMM (Flexible Quality of service Model for Mobile ad-hoc networks) [5], and DS-SWAN (Differentiated Services-SWAN) [17]. DS-SWAN supports end-to-end QoS in ad-hoc networks connected to fixed DiffServ domains. The QoS proposal addressing the integration of ad-hoc networks and infrastructure networks was also deployed within the confines of Daidalos I project [1] and published in [7]. The work presented in this paper continues the concept of ad-hoc and infrastructure networks integration with complete end-to-end QoS support for four traffic classes. Following Daidalos I architecture, the QoS model in the wireless ad-hoc network is based on the extended SWAN approach. The QoS solution developed in Daidalos I was simplified by exploiting IEEE 802.11e technology, therefore introducing QoS differentiation at layer 2. The ad-hoc QoS mechanism is integrated with the NSIS signaling protocols suite [10] in the infrastructure network, which provides more flexibility in end-to-end signaling supporting different resource management models, and makes possible to set up bidirectional reservation. For outof-path QoS signaling in infrastructure network a Diameter [11] protocol is used. Due to build-in security mechanisms it can be used for both inter and intra administrative domain signalling.

The paper is organized as follows. The proposed QoS architecture as well as the mobile node and gateway node schemes are presented in Chapter II. Chapter III contains the details about the SWAN protocol extensions for ad-hoc network integration including: signaling and dynamic regulation process, QoS differentiation and MAC layer measurements. The signaling protocols used in infrastructure are presented in Chapter IV. At the end of the paper, the conclusions are showed.

\section{Network QoS Architecture}

The QoS architecture presented in this paper is the continuation of work provided in IST Daidalos project [1] on QoS architecture in Next Generation Networks (NGN). The framework of NGN is recently under study by the most important standardization organization including International Telecommunication Union - Telecommunication Standardization Sector (ITU-T) Study Group 13 (SG13) and Focus Group NGN (FGNGN), and European Telecommunications Standard Institute (ETSI) TISPAN [8] initiative. Currently NGN standards groups become tightly coupled with mobile groups - SG13 and FGNGN with SG19, and TISPAN with 3GPP. The results of joint 
efforts can be found e.g. in [10], where integration of 3GPP QoS architecture in NGN is proposed.

In comparison to Daidalos I the main changes in the QoS architecture are:

1. supporting new signalling protocols, adding more flexibility in session setup,

2. support of Local Mobility Management,

3. tighter integration of broadcast and multicast,

4. integration of terminal multihoming,

5. new QoS solutions for the access network including ad-hoc.

In this paper the focus is put on QoS mechanisms in ad-hoc networks and integration of ad-hoc with infrastructure.

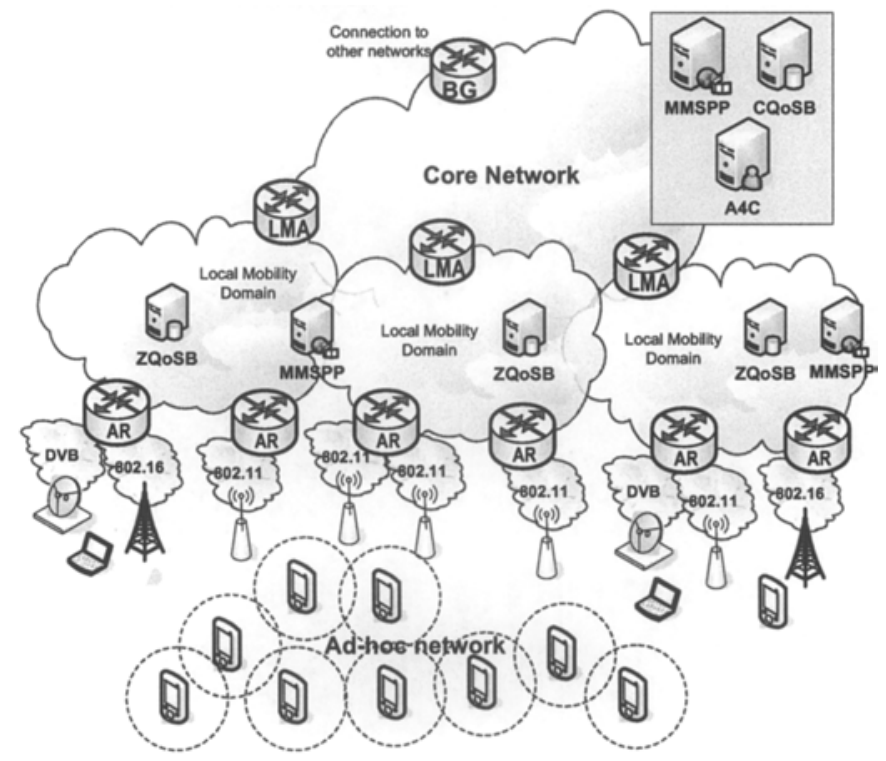

Fig. 1. QoS hierarchical architecture of a complete administrative domain.

In Fig. 1. the QoS architecture of a complete administrative domain is presented. The general QoS Daidalos II architecture follows the Daidalos I hierarchical network, where three main levels are distinguished. The top level makes up a core network, responsible for inter operator domains resource management and interconnecting second level Local Mobility Domains (LMD). The low level consists of access networks built in different technologies. The most important access network types from Daidalos point of view are IEEE 802.11 including ad-hoc and infrastructure mode, 802.16, DVB, and WCDMA.

In the Daidalos architecture, QoS resource management is divided into several areas, according to the structure of the network. The hierarchical architecture guarantees scalability and makes possible per-flow resource management in wireless access where scarce radio resources should be managed effectively. Whilst in the core network per aggregate traffic management using DiffServ [12] model of resource management is provided.

The mobility scheme deployed in the project is very important for QoS architecture due to its impact on QoS network structure. The mobility management is divided on 
Global Mobility Management where MIPv6 is used and Local Mobility Management. The protocol supporting local mobility LMP (Local Mobility Protocol) is designed in Daidalos, and is based on concepts proposed by IETF NetLMM [13], a hierarchical mobile IP [14] and the IEEE 802.21 proposed standard [15]. The main mechanisms of LMP are implemented in the network, but some of L2 mechanisms, like IEEE 802.21 signalling, have to be deployed in mobile node (MN). To support QoS in LMD (Local Mobility Domain) the QoS solution must be tightly coupled with LMP.

Inside LMD a hand-over between access routers (ARs) does not require the change of CoA in $\mathrm{MN}$ - from network layer point of view the $\mathrm{MN}$ does not perform any $\mathrm{L} 3$ address reconfiguration of interface. The $\mathrm{MN}$ only re-associates to a new access point (AP) with the same IP address. The entity controlling this process in LMD is LMA (Local Mobility Anchor). LMD can integrate several heterogeneous access networks. Although from point of view of end-to-end signalling the session does not change, the QoS on links between LMA and ARs must be maintained; QoS in access network has also to be appropriately controlled. The main entity managing QoS in LMD is the ZQoSBr (Zone QoS Broker), which controls all routers in the domain. $\mathrm{ZQoSBr}$ is also a policy enforcement point of $\mathrm{A} 4 \mathrm{C}$ (Authentication, Authorization, Accounting, Auditing and Charging) subsystem, controlling access to the network by QoS mechanisms deployed in ARs. In ARs there are interfaces responsible for QoS management in access networks (AN). To unify the access to different access technology mechanism the RAL (Radio Access Layer) was designed.

The entity responsible for QoS requirements signalling for application using SIP protocol is MMSPP (Multimedia Service Provisioning Proxy). MMSPP is responsible for extracting QoS requirements from QoS extended SIP protocol and signaling application requests to $\mathrm{ZQoSBr}$.

To really make benefits of ad-hoc network, the integration with infrastructure and end-to-end QoS resource management is required. In Daidalos II architecture the interoperation between ad-hoc and infrastructure solution is provided in AR and MN. The physical interconnection between ad-hoc and infrastructure is performed in the AR. The interaction between ad-hoc and mechanisms independent of access network technology are performed in the QoS Client (QoSC) module in the MN. QoSC provides general QoS API for applications to reserve network QoS resources for application data flows, which is independent of access network technologies.

\subsection{Ad-hoc QoS Architecture}

The ad-hoc mobile node has a double role, acting as a host which produces and consumes application traffic, and acting as a router that forwards the traffic of other nodes. The mobile node needs to be able to retrieve the QoS parameters from the application characteristics, triggers the check for QoS resources along the ad-hoc path, and checks the available resources in its wireless medium. It can also classify and mark the packets according to its class, ensure QoS differentiation, mark ECN bits and detect ECN marked packets in the case of congestion (See Fig. 2a).

The retrieval of the application QoS parameters is performed by the QoS Client; the Ad-hoc QoS Controller checks for QoS resources along the ad-hoc path and the available resources in its wireless medium. This is performed through the interaction 
with the MAC Measurement Module. The classification and marking of the packets is addressed in the Classification and ( $\mathrm{Re}$ )-Marking module, and the QoS differentiation is realized by the hardware queues implemented in WLAN cards based on Atheros chipset (L2 differentiation) [9]. To address congestion situations in the ad-hoc network, the node has an ECN Marking module that obtains the congestion status information from the MAC Measurement module and marks the ECN bits to trigger the dynamic regulation of the flows.

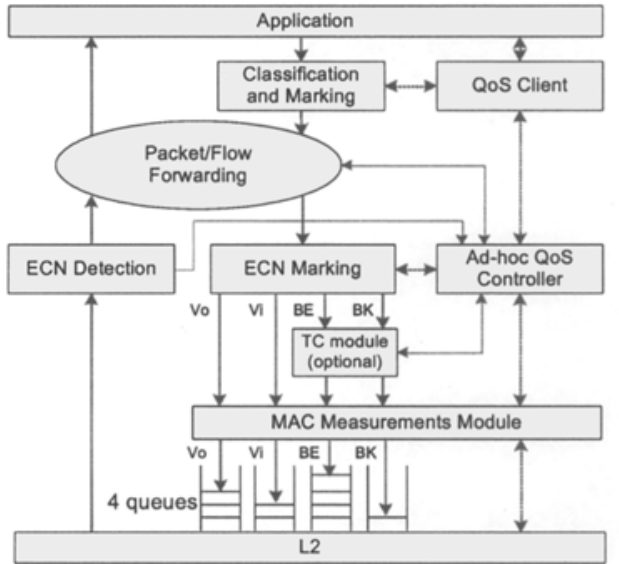

a)

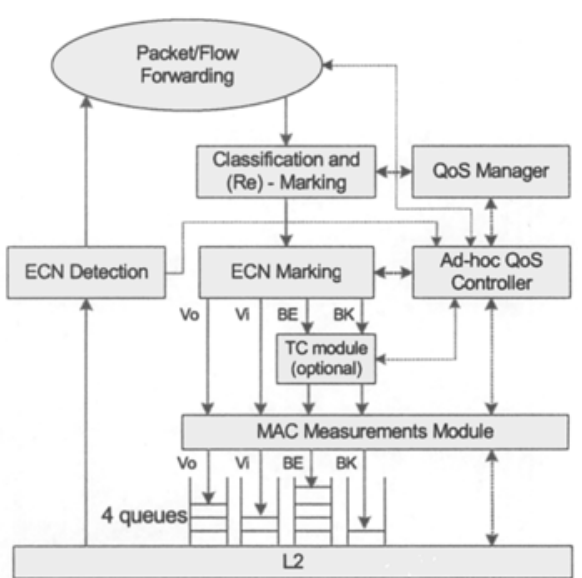

b)

Fig. 2. Mobile (a) and gateway (b) node schemes.

The gateway is able to support the same functionalities of the mobile nodes, but does not have interaction with the application signalling (since it works only at the IP layer and below) (see Fig. 2b). Instead, it needs to perform interoperation between the QoS signalling in the ad-hoc and the infrastructure side.

\subsection{Ad-hoc Network Technology Requirements}

WLAN cards based on IEEE $802.11 \mathrm{a} / \mathrm{b} / \mathrm{g} / \mathrm{e}$ standards are considered within MANET of Daidalos II project [2], [3]. The IEEE 802.11e standard with MAC layer QoS support should be used to perform L2 service differentiation. The IEEE $802.11 \mathrm{a} / \mathrm{b} / \mathrm{g}$ defines the number of physical layer types with maximum rates: $2 \mathrm{Mbps}$ in $2.4 \mathrm{GHz}$ band (IEEE 802.11), $11 \mathrm{Mbps}$ in 2.4 GHz band (IEEE 802.11b), $54 \mathrm{Mbps}$ in $2.4 \mathrm{GHz}$ band (IEEE 802.11 g), and $54 \mathrm{Mbps}$ in $5 \mathrm{GHz}$ band (IEEE 802.11a). The basic IEEE 802.11 standard was designed to operate in DCF (Distributed Coordination Function) and PCF (Point Coordination Function) modes. DCF is the fundamental access method used to support asynchronous data transmission on the best effort basis. IEEE $802.11 \mathrm{~b}$ with DCF mode was used in Daidalos I project. As previously referred, MAC Measurement Module (MMM) is responsible for QoS measurements (L2 frames). These measurements are reported to L3 QoS modules to perform L3 service differentiation. MAC layer measurements are crucial to obtain L3 QoS. 
IEEE 802.11e standard supports EDCA (Enhanced DCF Channel Access) which allows for L2 service differentiation. EDCA opens various parameters for service differentiation configuration, namely: $\mathrm{CW}_{\min }, \mathrm{CW}_{\max }$, AIFS, and TXOP. The EDCA is designed to provide differentiated, distributed channel accesses for frames with different priorities. The QoS support for EDCA can be achieved statistically by reducing the probability of medium access for lower priority traffic categories through the configuration of these parameters. Medium contention rules for EDCA are the same as in $802.11 \mathrm{DCF}$. Additionally, to achieve high medium utilization, the TXOP parameter was defined. TXOP is the time dedicated to the transmission of consecutive MAC frames of the same station. It is also recommended to always use RTS/CTS frames exchange before the data transmission to minimize the negative effect of hidden stations. L2 service differentiation can speed up the service differentiation process and allows to simplify L3 architecture.

\section{SWAN Signaling}

Similarly to Daidalos I, our enhanced SWAN concept has been chosen to build Daidalos II architecture due to the following features:

- it supports QoS negotiations and service differentiation for four different traffic classes, i.e., voice, video, best-effort, and background,

- it does not require any per-flow or aggregate state information in the intermediate nodes as it controls all traffic classes locally, with the use of MAC delay measurements,

- admission control is performed only at the source node with the use of the request/response probe which checks the available bandwidth on the path towards the destination.

When congestion/overload conditions occur (e.g., as a result of node mobility) the dynamic regulation of real-time sessions is possible with SWAN. In case a particular mobile node detects such a situation, it starts marking ECN bits in the IP header of real-time packets. When a destination node, monitoring the $\mathrm{ECN}$ bits, notices that they are marked, it sends a regulate message to the source so as to force it to reestablish the real-time session by sending a new probing request to the destination.

\subsection{QoS Signalling and Dynamic Regulation}

If the sender node is an ad-hoc mobile node, it sends an App_Sig Initiation message (message that corresponds to the signalling of the specific application start), and triggers a Probing Request message (Fig. 3). This request contains a Bottleneck Bandwidth (BB) field located in an IPv6 extension header that is updated in a hop-byhop basis with the minimum available bandwidth of the corresponding class in the path, and the Requested Bandwidth (RB) for the flow (the mobile node includes a QoS client module that maps the application QoS parameters into network QoS parameters). The Probing Request message is updated by every intermediate node in 
the ad-hoc network with the BB of the corresponding class (i.e., minimum bandwidth in the path). Every mobile node has a L2 measurement module that measures the occupied bandwidth and the delay corresponding to each class in the wireless medium. After receiving this message, the gateway checks the $B B$ and the $R B$ (optionally, it can also check the delay values). If the $\mathrm{BB}$ is larger than the RB, this means that the ad-hoc network has sufficient available bandwidth. It replies to the probing request with a Probe Response message with indication of the available bandwidth in the ad-hoc path. Otherwise, an error message is sent to the sender. In the case of available resources in the ad-hoc path, the mobile node sends a NSIS_reserve message, and the gateway checks for the authorisation in the infrastructure network, issuing a QoS request to the ZQoSBr.

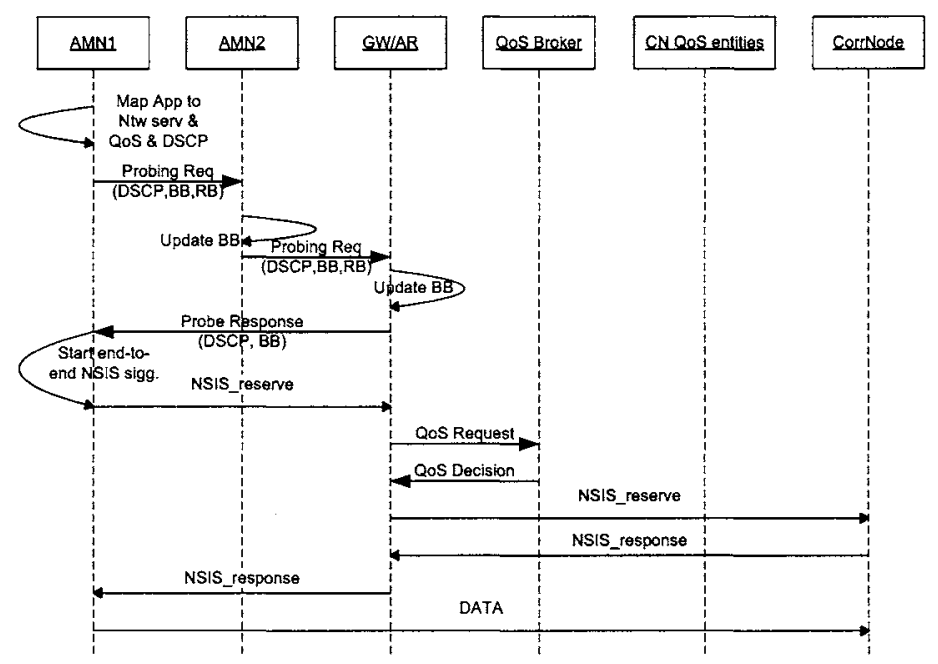

Fig. 3. Ad-hoc initiated session setup.

All out of path core signaling uses a Diameter protocol from the IETF DIME WG [11]. The gateway will request authorization from the ZQoSB that will make two verifications: first, if the user has permission for the service (communication with the $\mathrm{A} 4 \mathrm{C}$ ); second, if there are available resources for the request. In the case of a positive answer, it forwards the NSIS_reserve message to the receiver (correspondent node CorrNode) through the core network (CN) entities ( $\mathrm{CN}$ QoS entities). The correspondent node replies to the NSIS_reserve with an NSIS_response message and, if the session parameters are allowed in the two terminals, the setup process ends with an NSIS_response message to the sender node. If the sender is on the infrastructure side (Fig. 4), the GW recognizes the signalling message, asks the $\mathrm{ZQoSBr}$ for available resources in the infrastructure access network and sends a probe request message towards the receiver ad-hoc mobile node. If the probing process in the adhoc access network is successful the NSIS signalling is continued.

Fig. 5 depicts the case of dynamic regulation integrated in the infrastructure network. When an ad-hoc node detects an overioad condition in a specific class (i.e., the target bandwidth for the class is exceeded), this node starts marking ECN bits in 
packets of the affected class. The gateway monitors the ECN bits, and upon their detection, notifies the sources by sending Regulate messages. When a source receives a Regulate message it should perform application adaptation, or else, should re-start the probing process.

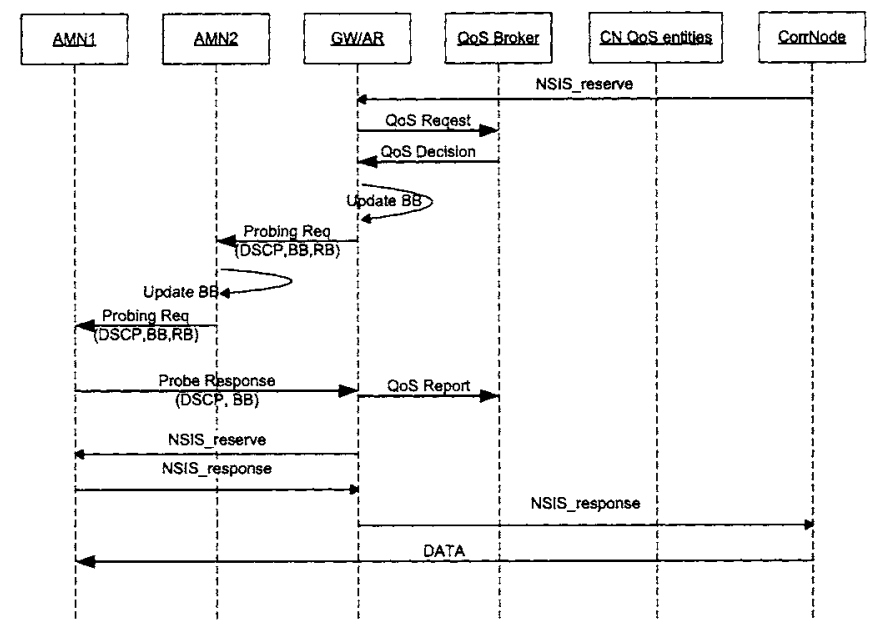

Fig. 4. Infrastructure initiated session setup.

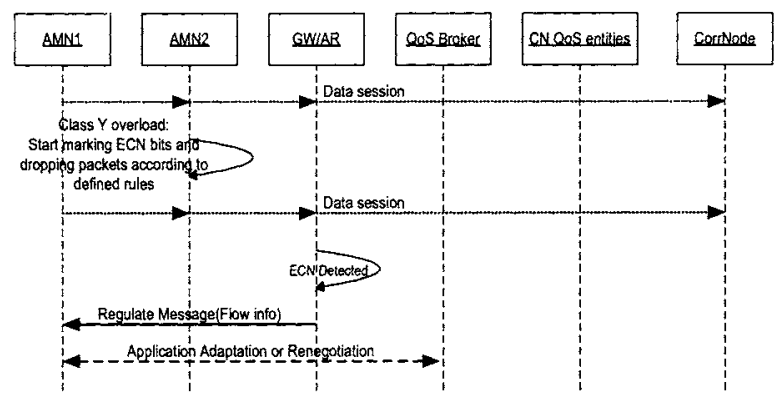

Fig. 5. Dynamic regulation.

\subsection{QoS Differentiation}

Service differentiation in the original SWAN concept assumes only two service classes, one specified for real-time UDP traffic and another one specified for besteffort TCP traffic. In Daidalos I the SWAN service level differentiation was expanded to four different traffic classes: voice, video, best effort, and background. Each of these classes has associated a set of service attributes. Request for bandwidth allocation for the desired traffic class is issued by a node that requests service at session setup time. The request can later be dynamically adjusted based on feedback 
from L2, especially for low priority classes. Bandwidth allocation for classes is controlled by rate limiters. It should be pointed that connection renegotiation can be required due to changing network conditions or excessive traffic passing through a mobile node in the ad-hoc network. The extended differentiation model in Daidalos I is composed by a classifier and by a cascade of priority schedulers, shapers and queues associated to each traffic class [7].

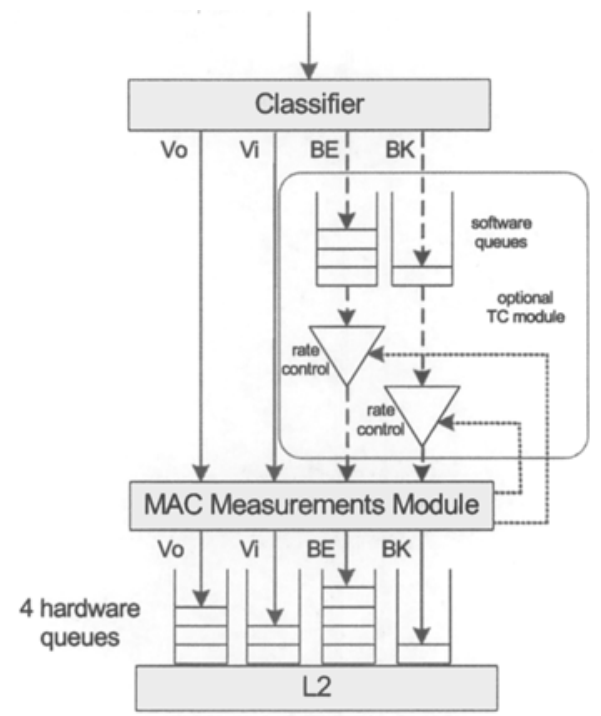

Fig. 6. The proposed Daidalos II ad-hoc service differentiation model.

The IEEE 802.11e WLAN cards possibilities (through hardware queues implementation) give an opportunity to realize L2 service differentiation. It should simplify L3 architecture and allows for faster L2 service differentiation. There is a plan to completely remove TC module (if L2 service differentiation will be sufficient) or use shapers only for two lower priority classes in new Daidalos II architecture. There is no sense to use shapers for conversational real-time services (voice) or streaming real-time services (video). It is better to renegotiate the new (lower) transmission rate (choose other voice or video codec type) for these real-time streams or discard new requests if we overload per class available bandwidth within these high priority classes than shape real-time streams. The shaping of real-time streams (voice or video) is unacceptable for most cases. The differentiation model needs to be complemented by per-class admission control of the two higher priority classes. An AIMD algorithm that has the MAC delay as feedback is proposed to control the shaping rate. The proposed service differentiation model is presented in Fig. 6.

\subsection{MAC Layer Measurements}

There is a need to obtain the MAC layer measurements information to assure a proper QoS level because fully distributed ad-hoc network is considered in this paper. Every mobile node has to perform some measurements in order to support admission control 
decisions and service differentiation functions (for two lowest priority classes only if L2 service differentiation will not be sufficient). The following parameters need to be measured: (1) Per class/overall delay - packet delay monitoring for four different classes/overall (from upper layer to the MAC layer and the time of the completion of RTS-CTS-DATA-ACK in EDCA; (2) Per-class/overall bandwidth utilization achieved by sensing the media and constructing periodic statistics about overall and per class (DSCP code) bandwidth occupancy; (3) Transmission rate - current WLAN card transmission rate (in case of IEEE $802.11 \mathrm{a} / \mathrm{b} / \mathrm{g}$ the stations communicate using transmission rates from 1 to $54 \mathrm{Mbps}$ ); (4) Number of stations - the estimation of the number of active stations in the neighbourhood to determine the contention and to evaluate the available bandwidth using current rate information.

\section{End-to-end Session Setup Signalling Strategies}

To support most typical applications including the legacy and multimedia ones in our QoS architecture, we consider three general signalling strategies: explicit request using NSIS that is described in subsection 3.1; extracting QoS needs from a SIP [16] mediated session initiation, and network triggered. The first two strategies are straightforward: in the NSIS scenario the terminal requests QoS on behalf of the application; in the SIP scenario (similar to the proposed in IMS or TISPAN), the QoS needs are inferred from the session description object. In the third strategy, the network handles the QoS reservation by acting on every starting flow. This means that every packet is classified according to a user profile residing in the network.

All three strategies can coexist in the network but which will be used depends on the application selection and MN capabilities. The first scenario will cover also QoS unaware legacy applications, for which the user will have utility which associates, in real-time, QoS service level with a certain application or flow. This request then triggers NSIS signaling with predefined parameters. In the following subsections the second and third signaling strategies are described shortly; the first scenario is presented in section 3 .

SIP Initiated End-to-end QoS Session Setup. The simplified QoS session setup for SIP with ad-hoc integration is presented in Fig. 7. The user application to reserve QoS resources signals its requirements to SIP-UA (SIP User Agent) module in MN. Then SIP-UA sends QOS request message to QoSClient module which forwards the request to ad-hoc QoS Controller. Then, the ad-hoc entities check the resources on the path to the infrastructure network by the probing procedure. After positive response SIP-UA sends SIP invite message to the MMSPP module in LMD. MMSPP authorizes the application request using signalling with $\mathrm{A} 4 \mathrm{C}$, and then signals for QoS requirements to the $Z Q \circ S B r$. ZQoSBr performs QoS resources admission control and after successful procedure sets up pre-reservation state for the session, and then sends the response result to the MMSPP. After successful response MMSPP continues SIP session set-up forwarding SIP invite to the corresponding node. After successful QoS reservation in the correspondent node network the MMSPP confirms to ZQoSBr the SIP session establishment. Then ZQoSBr reserves the QoS resources for the session and MMSP signals to SIP-UA the successful session set-up. 


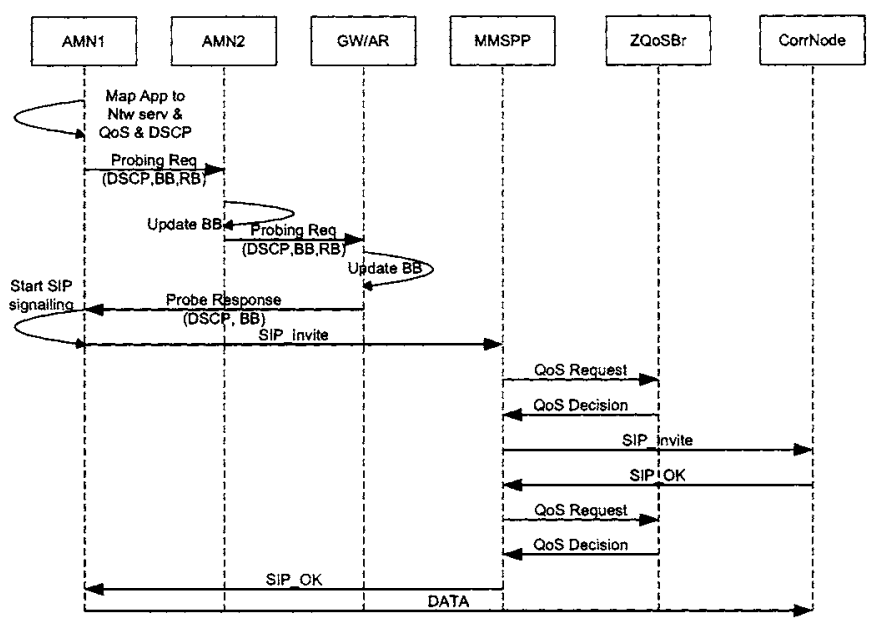

Fig. 7. SIP initiated session setup in ad-hoc.

Network Initiated QoS Reservations. A network initiated reservation is performed in the case when there are no QoS mechanisms implemented in Mobile Terminal. So this case does not support QoS in ad-hoc network due to assumption that the QoS mechanisms are not deployed in MNs. In that case, when AR detects a new flow it triggers QoS signalling with $\mathrm{ZQoSBr}$ to check the flow authorisation and QoS service level allocated to the flow in a user profile. To differentiate applications in the user service level agreement (SLA) has to be defined application flows filters and associated QoS service levels.

\section{Conclusions}

In the article the Daidalos II QoS architecture is described. The main focus is put on integration of ad-hoc networks with infrastructure ones to support end-to-end QoS for multimedia applications.

In the described architecture different schemes of signalling for session setup including SIP and NSIS protocols are supported. For DiffServ off-path signalling the Diameter with QoS extensions is proposed. The QoS mechanisms in ad-hoc networks, which make possible seamless integration with infrastructure, are described. The new IEEE 802.11e standard is used for L2 QoS differentiation. To the best authors knowledge the proposed QoS architecture for ad-hoc and infrastructure network integration exemplify one of the few complete solutions (the second after Daidalos I with four traffic classes support) for end-to-end QoS support in mobile ad-hoc networks.

This proposed architecture is being partially simulated in ns-2 and fully implemented in the Linux OS. Our future work concerns the validation of this QoS architecture through simulations and real experiments. 
Acknowledgments. The authors wish to thank the partners of the Daidalos II Consortium, in particular partners of WP2.4 and WP3.3 for their collaborative work.

Disclaimer. The work described in this paper is based on results of IST FP6 Integrated Project DAIDALOS. DAIDALOS receives research funding from the European Community's Sixth Framework Programme. Apart from this, the European Commission has no responsibility for the content of this paper. The information in this document is provided as is and no guarantee or warranty is given that the information is fit for any particular purpose. The user thereof uses the information at its sole risk and liability.

\section{References}

1. Daidalos IST Project: "Designing Advanced Interfaces for the Delivery and Administration of Location independent Optimised personal Services". (FP6-2002-IST-1506997)

2. IEEE 802.11 Standard for Wireless LAN: Medium Access Control (MAC) and Physical Layer (PHY) Specification, New York, IEEE Inc., 1999.

3. IEEE 802.11 e: MAC Quality of Service Enhancements, New York, IEEE Inc, Nov. 2005

4. Gahng-Seop Ahn, A. T. Campbell, A. Veres, and Li-Hsiang Sun: Supporting Service Differentiation for Real-Time and Best-Effort Traffic in Stateless Wireless Ad-Hoc Networks (SWAN). In IEEE Trans. on Mobile Comp., vol. 1, no. 3, pp. 192-207, July 2002

5. Hannan Xiao, Winston K.G. Seah, Anthony Lo, and Kee Chaing Chua: A Flexible Quality of Service Model for Mobile Ad-Hoc Networks. In Proceedings of the IEEE Vehicular Technology Conference, Tokyo, Japan, pp. 445-449, May 2000

6. S. B. Lee et al., INSIGNIA: An IP-Based Quality of Service Framework for Mobile AdHoc Networks J. Parallel and Distrib. Comp., Special issue on Wireless and Mobile Computing and Communications, vol. $60 \mathrm{n}^{\circ} 4$, pp. 374-406, Apr 2000

7. S. Crisóstomo, S. Sargento, M. Natkaniec, N. Vicari, A QoS Architecture Integrating Mobile Ad-Hoc and Infrastructure Networks - Workshop on Internet Compatible QoS in Ad-Hoc Wireless Networks, Egypt, 3-6 Jan 2005

8. A. Głowacz, M. Natkaniec, S. Sargento, S. Crisostomo: MAC Layer Measurements for Supporting QoS in IEEE 802.11 Ad-Hoc Networks - Workshop to QoS, held in conjunction with the Networking Conference, Coimbra, Portugal, 15-19 May 2006

9. MADWiFi - Multiband Atheros Driver for WiFi, http://madwifi.org

10. IETF, Next Steps in Signaling (nsis) charter: http://www.ietforg/html.charters/nsischarter.html

11. IETF, Diameter Maintenance and Extensions charter: http://www.ietf.org/html.charters/dime-charter.html

12. S. Blake (ed) et al., An Architecture for Diff. Services, IETF RFC 2475, Dec. 1998

13. Soliman et al., Hierarchical Mobile IPv6 Mobility Management (HMIPv6), RFC4140, IETF, august 2005

14. IETF, Network-based Localized Mobility Management charter: http://ww.ietf.org/htmi.charters/netlmm-charter.html

15. Draft Standard for Local and Metropolitan Area Networks: Media Independent Handovers Services (Draft .00). IEEE, March 2006

16. J. Rosenberg, et al, "SIP: Session Initiation Protocol", RFC 3261 
17. M.C. Domingo, D. Remondo: Quality of Service Support in Wireless Ad Hoc Networks Connected to Fixed DiffServ Domains, Proc. IFIP TC6 9th International Conference on Personal Wireless Communications PWC 2004, Delft, The Netherlands, September 2004 